\title{
Two-Dimensional GaN: An Excellent Electrode Material Providing Fast Ion Diffusion and High Storage Capacity for Li-Ion and Na-Ion Batteries
}

Xiaoming Zhang, Lei Jin, Xuefang Dai, Guifeng Chen, and Guodong Liu*

School of Materials Science and Engineering, Hebei University of Technology, Tianjin 300130, PR China. E-mail:gdliu1978@126.com

\begin{abstract}
Identifying applicable anode materials is a significant task for $\mathrm{Li}$ - and Na-ion battery (LIB and NIB) technologies. We propose the GaN monolayer (2D GaN) can be a good anode candidate. The GaN monolayer manifests stable Li/Na adsorption and inherently low theoretical voltages. Most excitingly, both high storage capacity and extremely fast Li/Na diffusion can be simultaneously realized in the GaN monolayer. For $\mathrm{Li}$, the storage capacity and diffusion barrier is $938 \mathrm{~mA} \mathrm{~h} \mathrm{~g}^{-1}$ and $80 \mathrm{meV}$, respectively. And the values for $\mathrm{Na}$ are $625 \mathrm{~mA} \mathrm{~h} \mathrm{~g}^{-1}$ and $22 \mathrm{meV}$. Comparing with known 2D anode materials with similar scale of ion diffusion barriers, the GaN monolayer almost possesses the highest Li/Na storage capacity discovered to date. Our work suggests that the $2 \mathrm{D} \mathrm{GaN}$ is a prospective anode material offering fast ion diffusion and high storage capacity.
\end{abstract}

Keywords: Li-ion batteries; Na-ion batteries; Anode materials; 2D GaN; First-principles 


\section{Introduction}

The Li-ion batteries (LIBs) have been intensively used nowadays. The success of LIBs mainly benefits from their excellent energy conversion efficiency and good storage capacity. ${ }^{1-5}$ Nevertheless, the development of LIBs has currently faced increasing challenge due to the naturally limited lithium resource and their high production cost. ${ }^{6-8}$ As a result, non-Li-ion batteries have emerged in recent years. ${ }^{9-15}$ Among them, Na-ion batteries (NIBs) have attracted the most attentions. ${ }^{9,10}$ This arises from that the sodium has relatively low cost. For LIBs and NIBs, the electrochemical performance is the most dependent on their electrode materials; thus developing good electrode materials is one of major focuses in current battery technologies. Two-dimensional (2D) materials have offered great potential as ion battery electrodes, because their fully exposed surfaces are believed to provide fast ion diffusion and the maximum ion insertion channels. ${ }^{16-18} \mathrm{Up}$ to date, several families of 2D materials, such as graphene, ${ }^{19-21}$ MXenes, ${ }^{22-28}$ transition-metal dichalcogenides (TMDCs), ${ }^{29-32}$ transition-metal dinitrides (TMDNs), ${ }^{33}$ borophenes, ${ }^{34-36}$ and others ${ }^{37-40}$ have been identified as LIBs and NIBs electrodes. For battery electrode materials, the ion diffusion speed and the storage capacity are two of the most crucial indicators for the electrode performances. However, most known 2D electrode materials can only achieve high performance on one of these indicators. 2D anode materials, for example, $\mathrm{Mo}_{2} \mathrm{C},{ }^{24,25} \mathrm{Nb}_{2} \mathrm{C}^{26}$ and $\mathrm{Ti}_{3} \mathrm{C}_{2}{ }^{23}$ are proposed to offer very fast $\mathrm{Li} / \mathrm{Na}$ diffusion speed but have low or moderate storage capacities; silicence $^{41}$ and $\beta_{12} / \chi_{3}$ Borophene ${ }^{35}$ show extremely high Li/Na storage capacities but have poor ion diffusion speed. Thus there is urgent need to explore excellent electrode materials which can offer fast ion diffusion and high storage capacity simultaneously.

As a well-known semiconductor, GaN has been widely used for variable applications. ${ }^{42}$ Unlike traditional 2D materials, it is previously quite a challenge to prepare freestanding 2D GaN by routine exfoliation technologies, because its bulk phase naturally crystallizes in the non-layered wurtzite structure. ${ }^{43}$ Nevertheless, large area 2D 
GaN has successfully synthesized quite recently, utilizing the graphene encapsulation method. ${ }^{44}$ Freestanding 2D GaN is experimentally characterized to stabilize in the buckled structure, with surface fully passivated by hydrogen atoms. Its buckled structure, as well as the stability of the $2 \mathrm{D} \mathrm{GaN}$, have been further verified by following

computations. ${ }^{45-47}$ Especially, it is predicted that, when the $2 \mathrm{D} \mathrm{GaN}$ is in the monolayer state, it would show interesting physical properties. ${ }^{48}$ Beyond these exotic properties, 2D $\mathrm{GaN}$ is also potential to be a superior battery electrode material, considering the fact that 2D GaN is environmentally friendly and possesses a very low mass density. However, 2D GaN has not been investigated on this aspect yet.

In this work, by using the GaN monolayer as the prototype system, we perform first-principles computations to investigate the feasibility of $2 \mathrm{D} \mathrm{GaN}$ as the battery electrode material. The detailed computational methods are provided in the Supporting Information. We fully study Li/Na adsorption and diffusion processes. We find that the GaN monolayer shows quite excellent performances as the battery anode material. Especially, the diffusion barriers for $\mathrm{Li}$ and $\mathrm{Na}$ ions are greatly lower than known 2D electrode materials. This manifests extremely fast ion diffusion on the GaN monolayer. Moreover, the semiconducting $\mathrm{GaN}$ become metallic after adsorptions with $\mathrm{Li}$ and $\mathrm{Na}$ atoms, with quite high ion storage capacities. Our results indicate that, the GaN monolayer can simultaneously possess good storage ability and fast ion diffusion as a battery electrode material.

\section{Structure of 2D GaN}

Bulk GaN crystallizes in the tetrahedral-coordinated wurtzite structure, so its monolayer would naturally possess unsaturated dangling bonds when directly cleaved from the bulk. Former experiments and computations ${ }^{44,48}$ have verified that, the GaN monolayer is the most thermodynamically stable when its surfaces are fully passivated by hydrogen. We have also performed formation-energy computations to show the energy preference of the 
GaN monolayer. The formation-energy of H-passivated GaN monolayer yields to be 0.91 $\mathrm{eV}$ lower than the unpassivated one, which agrees well with previous results. ${ }^{44,48}$

In Fig. 1(a)-(c), we show the $4 \times 4 \times 1$ supercell of the optimized GaN monolayer. It manifests a buckled structure under hydrogen passivation. The optimized in-plane lattice parameter for the unit cell is $3.17 \AA$, which is only $0.63 \%$ underestimated comparing its bulk phase (3.19 $\AA$ ). ${ }^{49}$ The relaxed bonding lengths for Ga-N, Ga-H, and N-H are found to be $2.01 \AA, 1.80 \AA$, and $1.50 \AA$, respectively.

\section{Adatom adsorption on GaN}

To begin with, we indentify the most favorable adatom adsorption site on the GaN monolayer. As shown in Fig. 1(a) and (c), for the top and the bottom surfaces of GaN, we have considered four high symmetry adsorption sites (denoted as T1-T4, and B1-B4, respectively). The adsorption energies for these sites are calculated by using the formula:

$$
E_{\mathrm{Ad}}=E_{\mathrm{LiNa}+\mathrm{GaNH}_{2}}-E_{\mathrm{GaNH}_{2}}-E_{\mathrm{L} / \mathrm{Na}}
$$

In formula (1), $E_{\mathrm{Ad}}$ is the adsorption energy. $E_{\mathrm{L} / \mathrm{Na}+\mathrm{GaNH}_{2}}\left(E_{\mathrm{GaNH}_{2}}\right)$ is the energy of the GaN supercell after (before) adatom adsorptions. $E_{\mathrm{Li} / \mathrm{Na}}$ denotes the energy for per $\mathrm{Li} / \mathrm{Na}$ atom.

Figure 2(a) and (b) show the calculated Li/Na adsorption energies. One can observe that their adsorption energy curves show quite similar features. First, GaN shows positive adsorption energies for all the bottom surface sites while has negative adsorption energies for all the top surface sites. This shows that the adsorptions prefer to occur on the top surface of GaN. Second, among the top adsorption sites (T1-T4), T2 and T3 sites have the same adsorption energy (because adatoms at T3 site would automatically shift to the $\mathrm{T} 2$ site), which is lower than those at T1 and T4 sites. Therefore, the adsorption at the T2 site is the most energetically favorable. From our computations, the adsorption energy is $-0.46 \mathrm{eV}(-0.25 \mathrm{eV})$ for per $\mathrm{Li}(\mathrm{Na})$ atom. These values are comparable with some typical 
2D electrode materials. For instance, in terms of adsorption energies, we have for $\mathrm{Sr}_{2} \mathrm{~N}$ $\left(-0.13 \mathrm{eV}\right.$ for $\left.\mathrm{Na}^{27}\right), \mathrm{V}_{2} \mathrm{C}\left(-0.16 \mathrm{eV}\right.$ for $\left.\mathrm{Li}^{22}\right), \mathrm{Mo}_{2} \mathrm{C}$ (about $-0.43 \mathrm{eV}$ for $\mathrm{Li}^{24}$, about -0.64 $\mathrm{eV}$ for $\left.\mathrm{Na}^{24}\right)$, and $\mathrm{MoS}_{2}\left(-0.60 \mathrm{eV}\right.$ for $\left.\mathrm{Li}^{30}\right)$. In this sense, $\mathrm{GaN}$ is promising for 2D electrode materials.

Here we study possible charge transfers during adsorptions. We find that $\mathrm{Li}$ and $\mathrm{Na}$ atoms transfer their most electrons to the GaN monolayer. The results are shown in Table 1. The amounts of transferred charges are larger than $0.8 e$ for both adatoms. Such charge transfers indicate that these adatoms are indeed chemically adsorbed and form chemical compounds with the GaN monolayer.

We continue to study the conductivity of GaN after Li/Na adsorptions. For this point, we compare the electronic structure of the GaN monolayer before and after adsorptions. In Fig. 3, we display the corresponding total density of states (DOS) and the optimized atomic configurations. One can find that, although the pristine $\mathrm{GaN}$ monolayer is a semiconductor, it would become metallic during the adsorptions. This is a quite desired condition as a battery electrode candidate. The sufficient charge transfer from adatoms is responsible for this semiconductor-to-metal transition. Similar phenomenon has also been observed in other semiconducting electrode materials such as silicene, ${ }^{41}$ phosphorus $^{37,38,50,51}$ and GeS sheets ${ }^{39,52}$.

\section{Adatom diffusion on GaN}

Next, we estimate the rate performance of the GaN monolayer when using as LIBs and NIBs electrodes. For this point, we have considered three migration paths (P1, P2, P3) between neighbored T2 sites. The pathways and calculated diffusion profiles are shown in Fig. 4. One observes that the energy barrier for P2 is the lowest among the three paths, which is $79 \mathrm{meV}$ for $\mathrm{Li}$ and $22 \mathrm{meV}$ for $\mathrm{Na}$. Further calculations show that the $\mathrm{Li} / \mathrm{Na}$ diffusion barrier can be even lower with increasing the adatom coverage. We have calculated layer-dependent ion diffusion barrier on the GaN monolayer. Based on our 
computations, the $\mathrm{Li}(\mathrm{Na})$ diffusion barrier drops to $43 \mathrm{meV}$ (16 meV) for one-layer-coverage and $37 \mathrm{meV}(11 \mathrm{meV})$ for two-layers-coverage. It is worth noting that, these diffusion barriers are lower than most 2D anode materials. This indicates extremely fast $\mathrm{Li} / \mathrm{Na}$ diffusion on the GaN monolayer. We will make detailed discussions on this aspect in section 6 .

\section{Li/Na storage capacity on GaN}

In this section, we focus on the $\mathrm{Li} / \mathrm{Na}$ storage capacity on GaN. The adsorption energies are computed layer-by-layer. Here the computations are performed on the $4 \times 4 \times 1$ supercell of the GaN monolayer. The half-cell reactions can be described as:

$$
\mathrm{GaNH}_{2}+x \mathrm{M}^{+}+x e^{-} \longleftrightarrow \mathrm{MGaNH}_{2} \quad \mathrm{M} / \mathrm{M}^{+}(\mathrm{M}=\mathrm{Li}, \mathrm{Na})
$$

Therefore, the average OCV $\left(V_{\text {ave }}\right)$ can be calculated follows:

$$
V_{\mathrm{ave}}=\left(E_{\mathrm{GaNH}_{2}}+x E_{\mathrm{M}}-E_{\mathrm{MGaNH}_{2}}\right) / x e
$$

where $E_{\mathrm{GaNH}_{2}}, E_{\mathrm{MGaNH}_{2}}$ and $E_{\mathrm{M}}$ have been defined above. Here $x$ is the number of adatoms. The voltage profile, which is the plot of $V_{\text {ave }} v s$. Li/Na concentration, can reflect the sequence of the layer-by-layer $\mathrm{Li} / \mathrm{Na}$ adsorptions on the $\mathrm{GaN}$ monolayer, since each layered adsorption would show a plateau in the voltage profile. To obtain the voltage profile, we first calculate the layer-resolved adsorption energy $\left(E_{\text {layer-ave }}\right)$, which follows:

$$
E_{\text {layer-ave }}=\left(E_{\mathrm{M}_{16 \mathrm{n}} \mathrm{Ga}_{16} \mathrm{~N}_{16} \mathrm{H}_{32}}-16 E_{\mathrm{M}}-E_{\mathrm{M}_{16(\mathrm{n}-1)} \mathrm{Ga}_{16} \mathrm{~N}_{16} \mathrm{H}_{32}}\right) / 16
$$

For the calculated $E_{\text {layer-ave }}$, the values of Li yield to be: $-0.34 \mathrm{eV}$ of the first layer, -0.19 $\mathrm{eV}$ of the second layer, $-0.08 \mathrm{eV}$ of the third layer, $0.11 \mathrm{eV}$ of the fourth layer; and $\mathrm{Na}$ : $-0.21 \mathrm{eV}$ of the first layer, $-0.07 \mathrm{eV}$ of the second layer, $0.08 \mathrm{eV}$ of the third layer.

Figure 5 shows the Li/Na voltage profiles. For $\mathrm{Li}$, the calculated voltage is about 0.261-0.060 V. The three voltage plateaus corresponds to the 1st, the 2nd and the 3rd 
layer adsorptions of $\mathrm{Li}$ atoms. For $\mathrm{Na}$, the voltage is about $0.157-0.050 \mathrm{~V}$. It shows two effective plateaus which corresponds to the 1st and the 2nd layer of Na adsorptions. To be noted, the third plateau in Fig. 5(b) shows a negative value of voltage. This indicates the third layer of Na is unlikely to be adsorbed. For LIBs and NIBs, the average OCV for the GaN monolayer is $0.05-0.26 \mathrm{~V}$. These values are among those of typical anodes, for instance $0.12-0.20 \mathrm{~V}$ for graphite, ${ }^{30,53} 0.12-0.68 \mathrm{~V}$ for borophene, ${ }^{34,35} 0.09-0.23 \mathrm{~V}$ for $\mathrm{Ca}_{2} \mathrm{~N},{ }^{27} 0.14 \mathrm{~V}$ for $\mathrm{Mo}_{2} \mathrm{C},{ }^{24,25}$ and $0.06 \mathrm{~V}$ for $\mathrm{Nb}_{2} \mathrm{C} .{ }^{26}$ Thus $\mathrm{GaN}$ is suitable to be applied as a LIB and NIB anode material.

According to the definition of equation (3), one can find that, the maximum capacity on the GaN monolayer corresponds to the $\mathrm{Li} / \mathrm{Na}$ concentration beyond which the $V_{\text {ave }}$ becomes negative. As shown in Fig. 5(a) and (b), the chemical stoichiometry for the maximum $\mathrm{Li}$ and $\mathrm{Na}$ adsorption is $\mathrm{Li}_{3} \mathrm{GaNH}_{2}$ and $\mathrm{Na}_{2} \mathrm{GaNH}_{2}$, respectively. Then we can calculate the maximum Li/Na storage capacity $\left(C_{\mathrm{m}}\right)$ by using the equation:

$$
C_{\mathrm{m}}=x_{\mathrm{m}} F / M_{\mathrm{GaNH}_{2}}
$$

Similar computations are also used in previous works. ${ }^{24-26,33}$ The calculated $\mathrm{Li}(\mathrm{Na})$ capacity is $938.1(625.4) \mathrm{mA} \mathrm{h} \mathrm{g}^{-1}$.

Here we examine the thermodynamic stability of the $\mathrm{Li}_{3} \mathrm{GaNH}_{2}$ and $\mathrm{Na}_{2} \mathrm{GaNH}_{2}$ system. Ab initio molecular dynamics (AIMD) simulations are adopted by using $4 \times 4 \times 1$ supercells of $\mathrm{Li}_{3} \mathrm{GaNH}_{2}$ and $\mathrm{Na}_{2} \mathrm{GaNH}_{2}$ at $323 \mathrm{~K}$. Each time step is chosen as 2 fs. Figure 6 shows the structure snapshots of initial and final states for $\mathrm{Li}_{3} \mathrm{GaNH}_{2}$ and $\mathrm{Na}_{2} \mathrm{GaNH}_{2}$. After 1500 steps, both $\mathrm{Li}_{3} \mathrm{GaNH}_{2}$ and $\mathrm{Na}_{2} \mathrm{GaNH}_{2}$ can retain their structures, indicating they can have good stability near the room temperature.

We also note that, several typical semiconductors BN, AlN and InN have the same structure with GaN. Their 2D counterparts are also promising to be synthesized by similar graphene encapsulation method. ${ }^{44}$ Based on equation (5), we can make an approximate estimation of the possible Li/Na capacity of BN, AlN and InN. If they can 
have the same $\mathrm{Li} / \mathrm{Na}$ adsorption abilities with the $\mathrm{GaN}$ monolayer, the Li capacity can be 3166.1 $\mathrm{mA} \mathrm{h} \mathrm{g}^{-1}$ for BN, $1915.1 \mathrm{~mA} \mathrm{~h} \mathrm{~g}^{-1}$ for AlN, and $609.6 \mathrm{~mA} \mathrm{~h} \mathrm{~g}^{-1}$ for InN. As to Na, the capacities are $2110.7 \mathrm{~mA} \mathrm{~h} \mathrm{~g}^{-1}$ for BN, $1276.7 \mathrm{~mA} \mathrm{~h} \mathrm{~g}^{-1}$ for AlN, and $406.4 \mathrm{~mA} \mathrm{~h} \mathrm{~g}^{-1}$ for InN, respectively. So this category of 2D materials is quite promising to serve as high capacity electrode materials.

\section{Comparison with typical 2D anode materials}

The diffusion barrier (which determines the rate capability) and the storage ability are quite crucial to battery electrodes. To get a comprehensive judgment of the electrode performance of the GaN monolayer, the parameters of some typical 2D anode materials for LIBs and NIBs are provided for comparison in Table 2 and Table 3.

For LIBs (see Table 2), one can find that the GaN monolayer possesses the second largest specific capacity among the listed ones. Although the capacity for the GaN monolayer is 0.3-1.1 times lower than $\beta_{12} / \chi_{3}$ Borophene, the Li diffusion can be 7.2-7.5 times faster in the GaN monolayer. When compared with commercial anode graphite, the GaN monolayer is 1.5 times higher in $\mathrm{Li}$ capacity and 4.7-14.2 times faster in $\mathrm{Li}$ diffusivity. Moreover, both Li storage capacity and diffusion performance in the GaN monolayer are much better than other anodes including phosphorene, $\mathrm{VS}_{2}$, and $\mathrm{GeS}$. It also worthies noticing that, some anode materials including $\mathrm{Mo}_{2} \mathrm{C}, \mathrm{Nb}_{2} \mathrm{C}$, and $\mathrm{Ti}_{3} \mathrm{C}_{2}$ possesses similar or a bit lower diffusion barriers, but their storage capacities (320-542 $\mathrm{mA} \mathrm{h} \mathrm{g}^{-1}$ ) are greatly lower than $\mathrm{GaN}\left(938 \mathrm{~mA} \mathrm{~h} \mathrm{~g}^{-1}\right.$ ). Therefore, GaN is quite suitable to be used as an LIBs anode with simultaneously realizing fast ion diffusion and excellent storage ability.

For NIBs, as shown in Table 3, both Na diffusion performance and storage ability in the GaN monolayer are much better than most typical $2 \mathrm{D}$ anode materials including $\mathrm{Ti}_{3} \mathrm{C}_{2}$, $\mathrm{GeS}, \mathrm{MoS}_{2}$, and $\mathrm{TiS}_{2}$. We also note that, $\mathrm{Nb}_{2} \mathrm{C}$ and $\mathrm{Mo}_{2} \mathrm{C}$ show similar diffusion barrier with the GaN monolayer, but their Na storage capacities are 1.3-3.7 times lower. For 
other anode materials listed in Table 3, including silicene/graphene, $\mathrm{MoN}_{2}$, and $\beta_{12} / \chi_{3}$ Borophene, one can observe that they show very high Na storage capacities but suffer quite poor Na diffusion ability (the diffusion barriers are as high as 110-560 meV); while the proposed GaN monolayer can be 5.0-25.5 times faster in Na diffusion. Remarkably, among available 2D anode materials with similar scale of Na diffusion barriers, the GaN monolayer almost possesses the highest Na storage capacity.

\section{Summary}

In summary, using the GaN monolayer as a prototype system, we have theoretically investigated the properties of the newly synthesized 2D GaN as potential battery electrodes. We find $\mathrm{Li}$ and $\mathrm{Na}$ atoms exhibit negative adsorption energies with sufficient charge transfers to the GaN monolayer, indicating their chemically stable adsorptions. The GaN monolayer becomes metallic after $\mathrm{Li}$ and $\mathrm{Na}$ adsorptions, which ensures good electronic conductivity as battery electrodes. The calculated average OCV is as low as $0.060 \mathrm{~V}$ for $\mathrm{Li}$ and $0.050 \mathrm{~V}$ for Na. This indicates $\mathrm{GaN}$ is suitable for the anode material. Remarkably, we find both the ion diffusion performance and storage capacity in the GaN monolayer are much higher than most known 2D anode materials. To be specific, the diffusion barrier is only $79 \mathrm{meV}$ and the capacity is $938 \mathrm{~mA} \mathrm{~h} \mathrm{~g}^{-1}$ for $\mathrm{Li}$, and those for $\mathrm{Na}$ are found to be $22 \mathrm{meV}$ and $625 \mathrm{~mA} \mathrm{~h} \mathrm{~g}^{-1}$. Our results suggest that both fast ion diffusion and excellent storage ability can be expected in the proposed GaN electrode.

\section{Acknowledgments}

This work is supported by the Special Foundation for Theoretical Physics Research Program of China (No. 11747152), Natural Science Foundation of Tianjin City (No.16JCYBJC17200), Research Project for High Level Talent of Hebei Province (No.A2017002020). 


\section{References}

(1) Oyama, N.; Tatsuma, T.; Sato, T.; Sotomura, T. Dimercaptan-Polyaniline Composite Electrodes for Lithium Batteries with High Energy Density. Nature 1995, 373, 598-600.

(2) Tarascon, J. M.; Armand, M. Issues and Challenges Facing Rechargeable Lithium Batteries. Nature. 2001, 414, 359-367.

(3) Dunn, B.; Kamath, H.; Tarascon, J.-M. Electrical Energy Storage for the Grid: a Battery of Choices. Science. 2011, 334, 928-935.

(4) Dubal, D. P.; Ayyad, O.; Ruiz, V.; Gomez-Romero, P. Hybrid Energy Storage: the Merging of Battery and Supercapacitor Chemistries. Chem. Soc. Rev. 2015, 44, $1777-1790$.

(5) Li, W.; Yang, Y.; Zhang, G.; Zhang, Y. W. Ultrafast and Directional Diffusion of Lithium in Phosphorene for High-Performance Lithium-Ion Battery, Nano Lett. 2015, 15,1691-1697.

(6) Tarascon, J.-M. Is Lithium the New Gold ? Nat. Chem. 2010, 2, 510.

(7) Goodenough, J. B.; Park, K.-S. The Li-Ion Rechargeable Battery: a Perspective. J. Am. Chem. Soc. 2013, 135, 1167-1176.

(8) Pan, H.; Hu, Y.-S.; Chen, L.Q. Room-Temperature Stationary Sodium-Ion Batteries for Large-Scale Electric Energy Storage. Energy Environ. Sci. 2013, 6, 2338-2360.

(9) Slater, M. D.; Kim, D.; Lee, E.; Johnson, C. S. Sodium-Ion Batteries. Adv. Funct. Mater. 2013, 23, 947-958.

(10) Eftekhari, A. Potassium Secondary Cell Based on Prussian Blue Cathode. J. Power Sources. 2004, 126, 221-228.

(11) Wessells, C. D.; Peddada, S. V.; Huggins, R. A.; Cui, Y. Nickel Hexacyanoferrate Nanoparticle Electrodes for Aqueous Sodium and Potassium Ion Batteries. Nano Lett. 2011, 11, 5421-5425.

(12) Singh, N.; Arthur, T. S.; Ling, C.; Matsui, M.; Mizuno, F. A High Energy-Density Tin Anode for Rechargeable Magnesium-Ion Batteries. Chem. Commun. 2013, 49, 
149-151.

(13) Ichitsubo, T.; Adachi, T.; Yagi, S.; Doi, T. Potential Positive Electrodes for High-Voltage Magnesium-ion Batteries. J. Mater. Chem. 2011, 21, 11764-11772.

(14) Liu, S.; Hu, J. J.; Yan, N. F.; Pan, G. L.; Li, G. R.; Gao, X. P. Aluminum Storage Behavior of Anatase $\mathrm{TiO}_{2}$ Nanotube Arrays in Aqueous Solution for Aluminum Ion Batteries. Energy Environ. Sci. 2012, 5, 9743-9746.

(15) Datta, D.; Li, J.; Shenoy, V. B. Defective Graphene as a High-Capacity Anode Material for Na- and Ca-Ion Batteries. ACS Appl. Mater. Interfaces. 2014, 6, 1788-1795.

(16) Liu, J.; Liu, X.-W. Two-Dimensional Nanoarchitectures for Lithium Storage. Adv. Mater. 2012, 24, 4097-4111.

(17) Zhang, H. Ultrathin Two-Dimensional Nanomaterials. ACS Nano 2015, 9, 9451-9469.

(18) Tan, C.; Cao, X.; Wu, X. J.; He, Q.; Yang, J.; Zhang, X.; Chen, J.; Zhao, W.; Han, S.; Nam, G.-H.; Sindoro, M.; Zhang, H. Recent Advances in Ultrathin Two-Dimensional Nanomaterials. Chem. Rev. 2017, 117, 6225-6331.

(19) Pollak, E.; Geng, B.; Jeon, K.-J.; Lucas, I. T.; Richardson, T. J.; Wang, F.; Kostecki, R. The Interaction of $\mathrm{Li}^{+}$with Single-Layer and Few-Layer Graphene. Nano Lett. 2010, 10, 3386-3388.

(20) Yamamoto, T.; Nohira, T.; Hagiwara, R.; Fukunaga, A.; Sakai, S.; Nitta, K.; Inazawa, S. Charge-Discharge Behavior of Tin Negative Electrode for a Sodium Secondary Battery Using Intermediate Temperature Ionic Liquid Sodium Bis(Fluorosulfonyl) Amide-Potassium Bis (Fluorosulfonyl) Amide. J. Power Sources. 2012, 217, 479-484.

(21) Tang, Q.; Zhou, Z.; Chen, Z. Graphene-Related Nanomaterials: Tuning Properties by Functionalization. Nanoscale. 2013, 5, 4541-4583.

(22) Hu, J.; Xu, B.; Ouyang, C.; Yang, S. A.; Yao, Y. Investigations on $\mathrm{V}_{2} \mathrm{C}$ and $\mathrm{V}_{2} \mathrm{CX}_{2}$ ( $\mathrm{X}=\mathrm{F}, \mathrm{OH})$ Monolayer as a Promising Anode Material for Li Ion Batteries from 
First-Principles Calculations. J. Phys. Chem. C. 2014, 118, 24274-24281.

(23) Er, D.; Li, J.; Naguib, M.; Gogotsi, Y.; Shenoy, V. $\mathrm{Ti}_{3} \mathrm{C}_{2}$ MXene as a High Capacity Electrode Material for Metal (Li, Na, K, Ca) Ion Batteries. ACS Appl. Mater. Interfaces. 2014, 6, 11173-11179.

(24) Sun, Q.; Dai, Y.; Ma, Y.; Jing, T.; Wei, W.; Huang, B. Ab Initio Prediction and Characterization of $\mathrm{Mo}_{2} \mathrm{C}$ Monolayer as Anodes for Lithium-Ion and Sodium-Ion Batteries. J. Phys. Chem. Lett. 2016, 7, 937-943.

(25) Çakır, D.; Sevik, C.; Gülserenc, O.; Peetersa, F. M. $\mathrm{Mo}_{2} \mathrm{C}$ as a High Capacity Anode Material: A First-Principles Study. J. Mater. Chem. A. 2016, 4, 6029-6034.

(26) Hu, J.; Xu, B.; Ouyang, C.; Zhang, Y.; Yang, S. A. Investigations on $\mathrm{Nb}_{2} \mathrm{C}$ Monolayer as Promising Anode Material for $\mathrm{Li}$ or Non-Li Ion Batteries from First-Principles Calculations. RSC Adv. 2016, 6, 27467-27474.

(27) Hu, J.; Xu, B.; Yang, S. A.; Guan, S.; Ouyang, C.; Yao, Y. 2D Electrides as Promising Anode Materials for Na-Ion Batteries from First-Principles Study. ACS Appl. Mater. Interfaces. 2015, 7, 24016-24022.

(28) Wan, Q.; Li, S.; Liu, J.-B. First-Principle Study of Li-Ion Storage of Functionalized $\mathrm{Ti}_{2} \mathrm{C}$ Monolayer with Vacancies. ACS Appl. Mater. Interfaces 2018, 10, 6369-6377.

(29) David, L.; Bhandavat, R.; Singh, G. $\mathrm{MoS}_{2} /$ Graphene Composite Paper for Sodium-Ion Battery Electrodes. ACS Nano. 2014, 8, 1759-1770.

(30) Jing, Y.; Zhou, Z.; Cabrera, C.; Chen, Z. Metallic VS 2 Monolayer: A Promising 2D Anode Material for Lithium Ion Batteries. J. Phys. Chem. C. 2013, 117, 25409-25413.

(31) Bhandavat, R.; David, L.; Singh, G. Synthesis of Surface-Functionalized $\mathrm{WS}_{2}$ Nanosheets and Performance as Li-Ion Battery Anodes. J. Phys. Chem. Lett. 2012, 3, 1523-1530.

(32) He, P.; Yan, M.; Zhang, G.; Sun, R.; Chen, L.; An, Q. Layered VS 2 Nanosheet-Based Aqueous Zn Ion Battery Cathode. Adv. Ener. Mater. 2017, 7(11), 1601920. 
(33) Zhang, X. M.; Yu, Z. M.; Wang, S.-S.; Guan, S.; Yang, H. Y.; Yao, Y. G.; Yang, S. A. Theoretical Prediction of $\mathrm{MoN}_{2}$ Monolayer as a High Capacity Electrode Material for Metal Ion Batteries. J. Mater. Chem. A. 2016, 4, 15224-15231.

(34) Jiang, H. R.; Lu, Z.; Wu, M. C.; Ciucci, F.; Zhao, T. S. Borophene: A Promising Anode Material Offering High Specific Capacity and High Rate Capability for Lithium-Ion Batteries. Nano Energy. 2016, 23, 97-104.

(35) Zhang, X. M.; Hu, J. P.; Cheng, Y. H.; Yang, H. Y.; Yao, Y. G.; Yang, S. A. Borophene as an Extremely High Capacity Electrode Material for Li-Ion and Na-Ion Batteries. Nanoscale. 2016, 8, 15340-15347.

(36) Naresh, K. J.; Rafael, B. A.; Vivekanand, S.; Rajeev, A. Borophane as a Benchmate of Graphene: A Potential 2D Material for Anode of Li and Na-Ion Batteries ACS Appl. Mater. Interfaces 2017, 9 (19), 16148-16158.

(37) Sun, J.; Lee, H.-W.; Pasta, M.; Yuan, H.; Zheng, G.; Sun, Y.; Li, Y.; Cui, Y. A Phosphorene-Graphene Hybrid Material as a High-Capacity Anode for Sodium-Ion Batteries. Nat. Nanotechnol. 2015, 10, 980-985.

(38) Li, W.; Yang, Y.; Zhang, G.; Zhang, Y.-W. Ultrafast and Directional Diffusion of Lithium in Phosphorene for High-Performance Lithium-Ion Battery. Nano Lett. 2015, 15, 1691-1697.

(39) Li, F.; Qu, Y.; Zhao, M. Germanium Sulfide Nanosheet: A Universal Anode Material for Alkali Metal Ion Batteries. J. Mater. Chem. A. 2016, 4, 8905-8912.

(40) Deng, S.; Wang, L.; Hou, T.; Li, Y. Two-Dimensional $\mathrm{MnO}_{2}$ as a Better Cathode Material for Lithium Ion Batteries. J. Phys. Chem. C. 2015, 119, $28783-28788$.

(41) Zhu, J. J. Udo Schwingenschlögl, Silicene for Na-Ion Battery Applications. 2D Mater. 2016, 3, 035012.

(42) Pimputkar, S.; Speck, J. S.; DenBaars, S. P.; Nakamura, S. Prospects for LED Lighting. Nat. Photonics. 2009, 3, 180-182.

(43) Dingle, R.; Sell, D. D.; Stokowski, S. E.; llegems, M. Absorption, Reflectance, and 
Luminescence of GaN Epitaxial Layers. Phys. Rev. B. 1971, 4, 1211-1218.

(44) Al Balushi, Z. Y.; Wang, K.; Ghosh, R. K.; Vilá, R. A.; Eichfeld, S. M.; Caldwell, J. D. ; Qin, X.; Lin, Y.-C.; DeSario, P. A.; Stone, G.; Subramanian, S.; Paul, D. F.; Wallace, R. M.; Datta, S.; Redwing, J. M.; Robinson, J. A. Two-Dimensional Gallium Nitride Realized Via Graphene Encapsulation. Nat. Mater. 2016, 15, 1166-1171.

(45) Lucking, M. C.; Xie, W.; Choe, D. H.; West, D.; Lu, T. M.; Zhang, S. B. Traditional Semiconductors in the Two-Dimensional Limit. Phys. Rev. Lett. 2018, 120, 086101.

(46) Zhang, H.; Meng, F. S.; Wu, Y. B. Two Single-Layer Porous Gallium Nitride Nanosheets: A First-Principles Study. Solid State Commun. 2017, 250, 18-22.

(47) Prete, M. S.; Mosca Conte, A.; Gori, P.; Bechstedt, F.; Pulci, O. Tunable Electronic Properties of Two-Dimensional Nitrides for Light Harvesting Heterostructures. Appl. Phys. Lett. 2017, 110, 012103.

(48) Sanders, N.; Bayerl, D.; Shi, G.; Mengle, K. A.; Kioupakis, E. Electronic and Optical Properties of Two-Dimensional GaN from First-Principles. Nano Lett. 2017, 17, $7345-7349$.

(49) Schulz, H.; Thiemann, K. H. Crystal Structure Refinement of AlN and GaN. Solid State Commun. 1977, 23, 815-819.

(50) Kulish, V. V.; Malyi, O. I.; Perssoncd, C.; Wu, P. Phosphorene as an Anode Material for Na-Ion Batteries: A First-Principles Study. Phys. Chem. Chem. Phys. 2015, 17, 13921-13928.

(51) Peng, Q.; Wang, Z.; Sa, B.; Wu, B.; Sun, Z. Blue Phosphorene/MS 2 (M = Nb, Ta) Heterostructures as Promising Flexible Anodes for Lithium-Ion Batteries. ACS Appl. Mater. Interfaces. 2016, 8, 13449-13457.

(52) Zhou, Y. G. MX (M = Ge, Sn; X = S, Se) Sheets: Theoretical Prediction of New Promising Electrode Materials for Li Ion Batteries. J. Mater. Chem. A. 2016, 4, 10906-10913.

(53) Prsson, K.; Hinuma, Y.; Meng, Y. S.; Van der Ven, A.; Ceder, G. Phys. Rev. B 2010, 
82, 125416.

(54) Tang, Q.; Zhou, Z.; Shen, P. Are MXenes Promising Anode Materials for Li Ion Batteries? Computational Studies on Electronic Properties and Li Storage Capability of $\mathrm{Ti}_{3} \mathrm{C}_{2}$ and $\mathrm{Ti}_{3} \mathrm{C}_{2} \mathrm{X}_{2}(\mathrm{X}=\mathrm{F}, \mathrm{OH})$ Monolayer. J. Am. Chem. Soc. 2012, 134, 16909-16916.

(55) Li,Y.; Wu, D.; Zhou, Z.; Cabrera, C. R.; Chen, Z. Enhanced Li Adsorption and Diffusion on $\mathrm{MoS}_{2}$ Zigzag Nanoribbons by Edge Effects: A Computational Study. J. Phys. Chem. Lett. 2012, 3, 2221-2227.

(56) Toyoura, K.; Koyama, Y.; Kuwabara, A.; Oba, F.; Tanaka, I. First-Principles Approach to Chemical Diffusion of Lithium Atoms in a Graphite Intercalation Compound. Phys. Rev. B 2008, 78, 214303.

(57) Ganesh, P.; Kim, J.; Park, C.; Yoon, M.; Reboredo, F. A.; Kent, P. R. C. Binding and Diffusion of Lithium in Graphite: Quantum Monte Carlo Benchmarks and Validation of Van Der Waals Density Functional Methods. J. Chem. Theory Comput. 2014, 10, 5318-5323.

(58) Mortazavi, M.; Wang, C.; Deng, J. K.; Shenoy, V. B.; Medhekar, N. V. Ab Initio Characterization of Layered $\mathrm{MoS}_{2}$ as Anode for Sodium-Ion Batteries. J. Power Sources. 2014, 268, 279-286.

(59) Yang, E.; Ji, E.; Jung, Y. Two-Dimensional Transition Metal Dichalcogenide Mono Layers as Promising Sodium Ion Battery Anodes. J. Phys. Chem. C. 2015, 119, 26374-26380. 
Table 1 Bader charge analysis for the GaN monolayer before and after $\mathrm{Li} / \mathrm{Na}$ adsorptions.

\begin{tabular}{|c|c|c|c|c|c|c|}
\hline & \multicolumn{7}{|c|}{ Average charge state } \\
\cline { 2 - 7 } & $\mathrm{Ga}$ & $\mathrm{H}(-\mathrm{Ga})$ & $\mathrm{N}$ & $\mathrm{H}(-\mathrm{N})$ & $\mathrm{Li}$ & $\mathrm{Na}$ \\
\hline $\mathrm{Ga}_{16} \mathrm{~N}_{16} \mathrm{H}_{32}$ & 1.739 & 1.297 & 6.356 & 0.608 & - & - \\
\hline $\mathrm{Ga}_{16} \mathrm{~N}_{16} \mathrm{H}_{32}+\mathrm{Li}$ & 1.741 & 1.313 & 6.336 & 0.621 & 0.137 & - \\
\hline $\mathrm{Ga}_{16} \mathrm{~N}_{16} \mathrm{H}_{32}+\mathrm{Na}$ & 1.742 & 1.303 & 6.328 & 0.631 & - & 0.170 \\
\hline
\end{tabular}


Table 2 Diffusion barrier and theoretical maximum capacity $\left(C_{\mathrm{M}}\right)$ of typical 2D anode materials for LIBs. The data of commercial graphite is also provided for comparison.

\begin{tabular}{|c|c|c|c|}
\hline Materials & $\begin{array}{l}\text { Diffusion barrier } \\
\text { (meV) }\end{array}$ & $C_{\mathrm{M}}\left(\mathrm{mA} \mathrm{h} \mathrm{g}^{-1}\right)$ & References \\
\hline 2D GaN & 79 & 938 & This work \\
\hline $\mathrm{Mo}_{2} \mathrm{C}$ & $15-43$ & $400-526$ & {$[24,25]$} \\
\hline $\mathrm{Nb}_{2} \mathrm{C}$ & 32 & 542 & [26] \\
\hline $\mathrm{Ti}_{3} \mathrm{C}_{2}$ & 70 & 320 & {$[23,54]$} \\
\hline Phosphorene & $130-760$ & $433-649$ & {$[37,38,50]$} \\
\hline $\mathrm{VS}_{2}$ & 220 & 466 & [30] \\
\hline $\mathrm{MoS}_{2}$ & 210 & 355 & {$[29,55]$} \\
\hline GeS & 236 & 256 & [39] \\
\hline$\beta_{12} / \chi_{3}$ Borophene & $600-660$ & $1240-1984$ & [35] \\
\hline $\mathrm{MoN}_{2}$ & 780 & 432 & [33] \\
\hline Graphite & $450-1200$ & 372 & {$[2,56,57]$} \\
\hline
\end{tabular}


Table 3 Diffusion barrier and theoretical maximum capacity $\left(C_{\mathrm{M}}\right)$ of typical 2D anode materials for NIBs.

\begin{tabular}{|c|c|c|c|}
\hline Materials & Diffusion barrier $(\mathrm{meV})$ & $C_{\mathrm{M}}\left(\mathrm{mA} \mathrm{h} \mathrm{g}^{-1}\right)$ & References \\
\hline $2 \mathrm{D} \mathrm{GaN}$ & 22 & 625 & This work \\
\hline $\mathrm{Nb}_{2} \mathrm{C}$ & 15 & 271 & {$[26]$} \\
\hline $\mathrm{Mo}_{2} \mathrm{C}$ & $19-25$ & 132 & {$[24,25]$} \\
\hline $\mathrm{Ti}_{3} \mathrm{C}_{2}$ & 96 & 319 & {$[23]$} \\
\hline $\mathrm{GeS}$ & 100 & 512 & {$[39]$} \\
\hline Silicene/graphene & 110 & $730-954$ & {$[41]$} \\
\hline MoS $_{2}$ & 280 & 146 & {$[58]$} \\
\hline TiS $_{2}$ & $>300$ & 339 & {$[59]$} \\
\hline$\beta_{12} / \chi_{3}$ Borophene & $330-340$ & $1240-1984$ & {$[35]$} \\
\hline MoN $_{2}$ & 560 & 864 & {$[33]$} \\
\hline
\end{tabular}




\section{Figures and captions:}

(a)

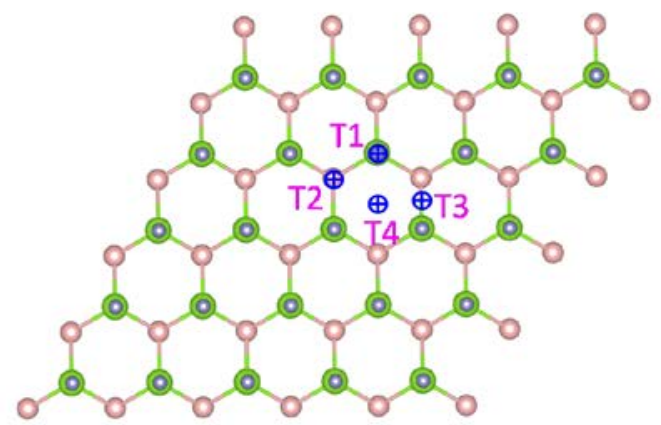

(b)

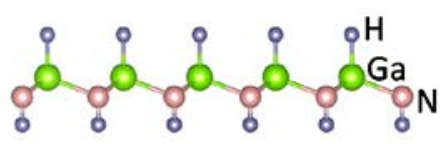

(c)

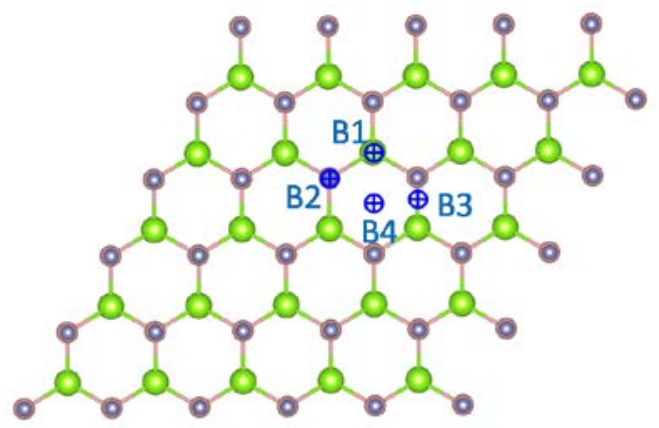

Fig. 1 Atomic structures of the GaN monolayer in (a) top, (b) side and (c) bottom views. In (a) and (c), T1-T4 and B1-B4 represent the possible adsorption sites on the top and the bottom surfaces of the GaN monolayer. 
(a)

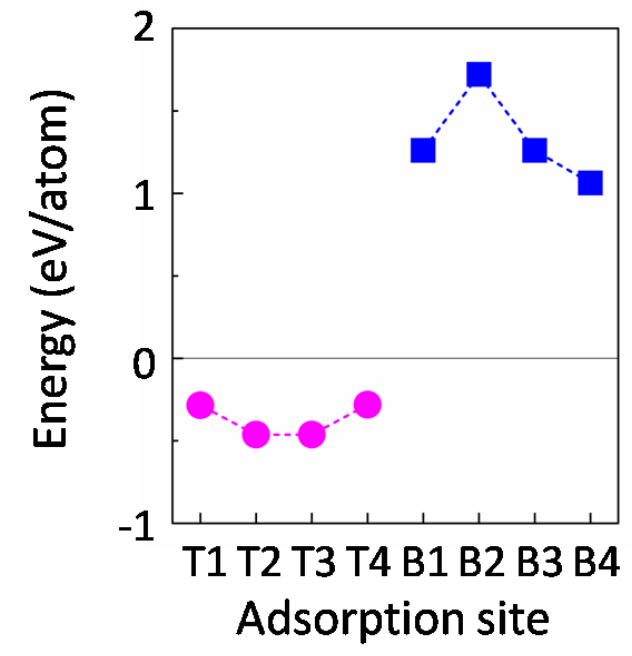

(b)

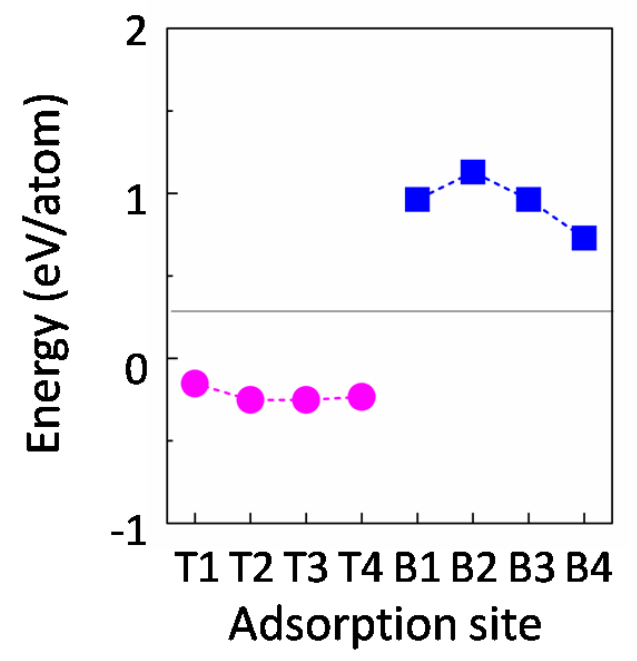

Fig. 2 Adsorption energies for (a) Li and (b) Na atoms on the GaN monolayer at different adsorption sites. 


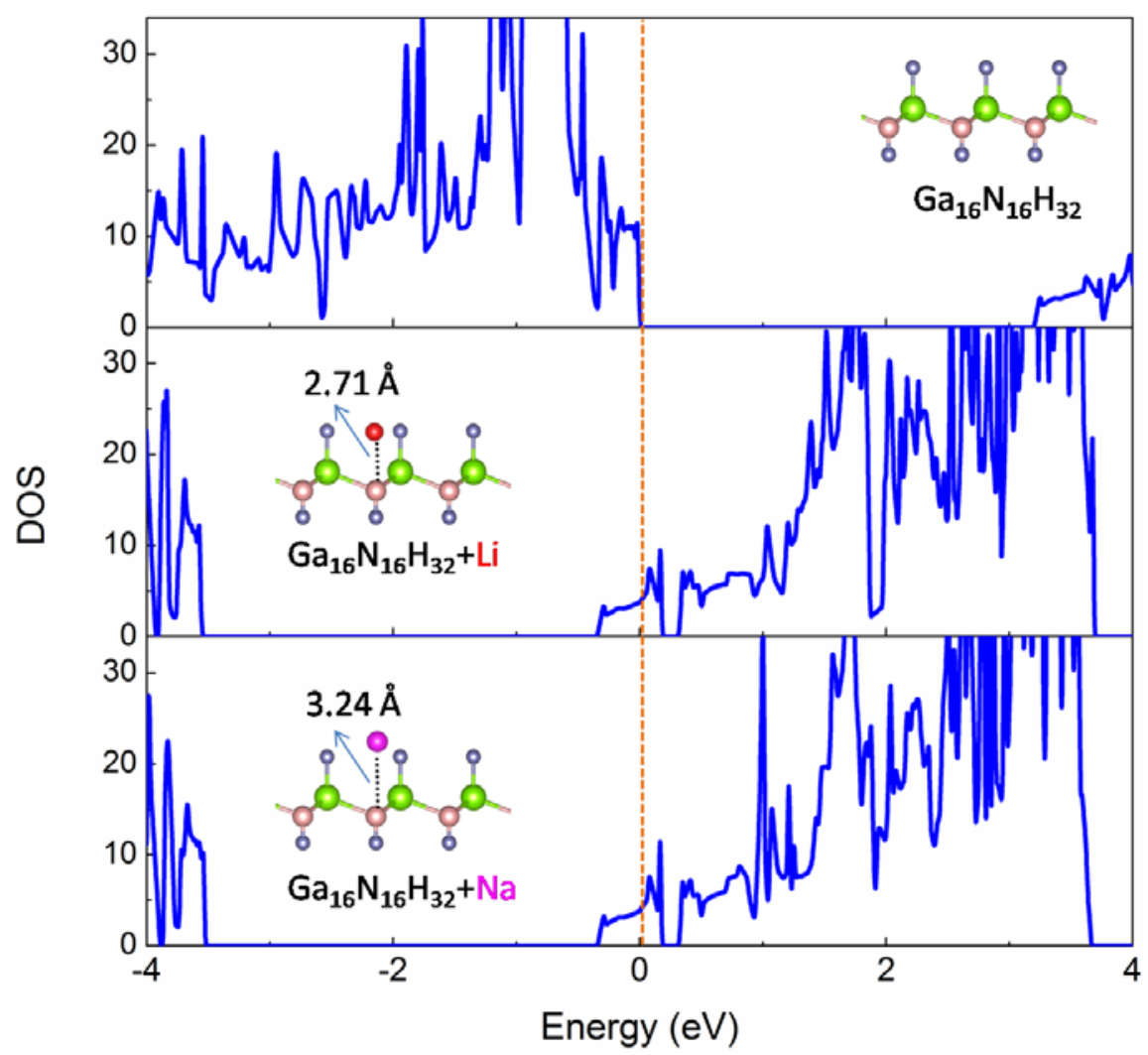

Fig. 3 Total density of states (DOS) of the GaN monolayer before and after $\mathrm{Li} / \mathrm{Na}$ adsorptions. Corresponding atomic structures are shown as the insets of the figure. 
(a)

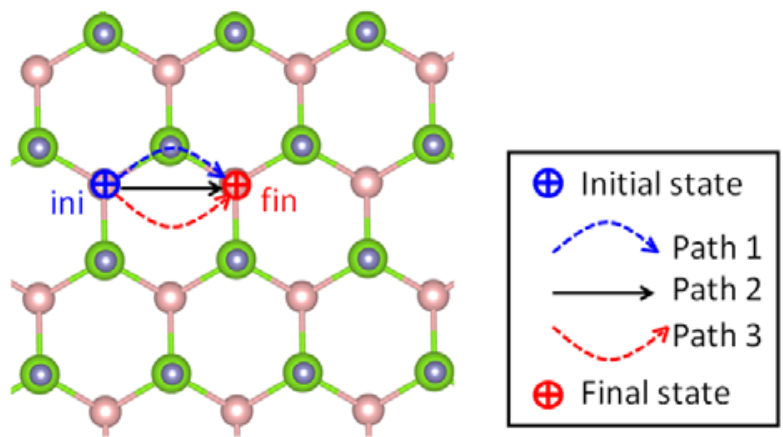

(b)

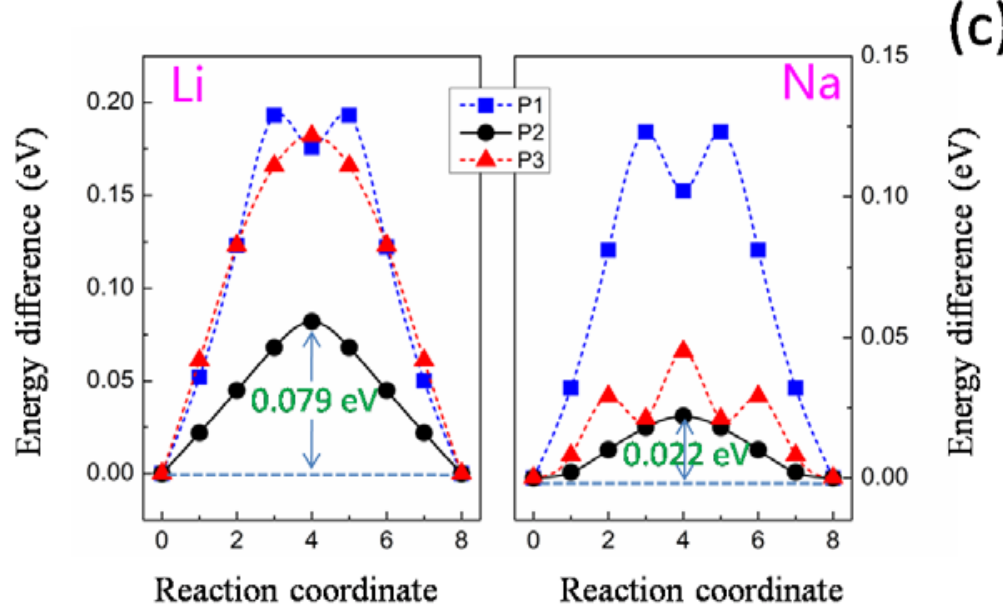

Fig. 4 (a) Possible Li/Na ion migration paths on the GaN monolayer. Corresponding diffusion barrier profiles for $\mathrm{Li}$ and $\mathrm{Na}$ are shown in (b) and (c), respectively. In (b) and (c), the minimum Li/Na diffusion barriers are labelled. 
(a)

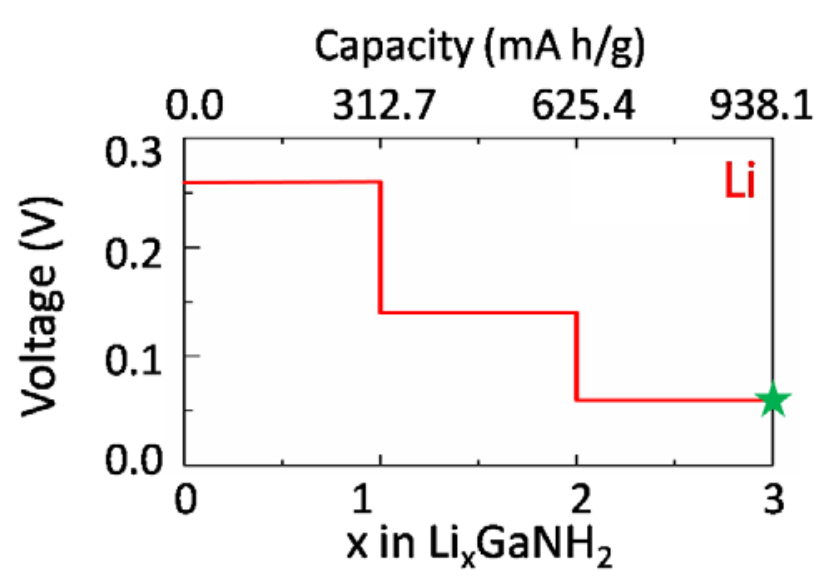

(b)

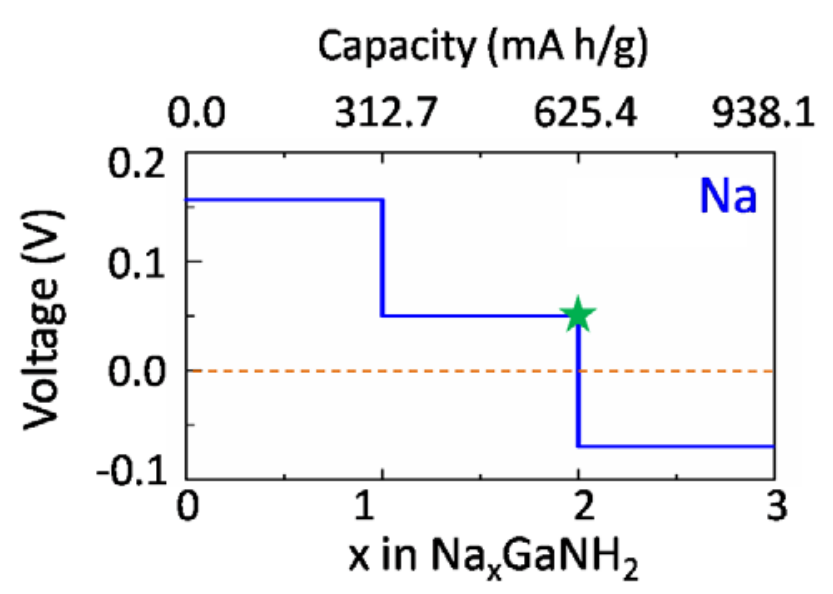

Fig. 5 The calculated voltage profiles and storage capacities for (a) Li and (b) $\mathrm{Na}$ on the GaN monolayer. The maximum Li and Na storage capacities are indicated by the stars in (a) and (b). 
(a)

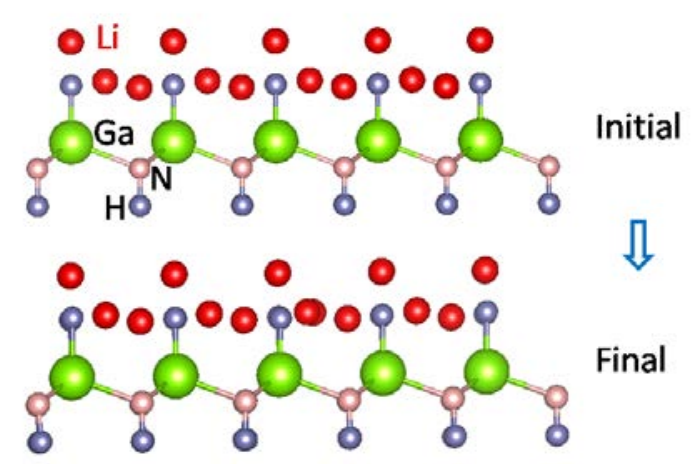

(b)

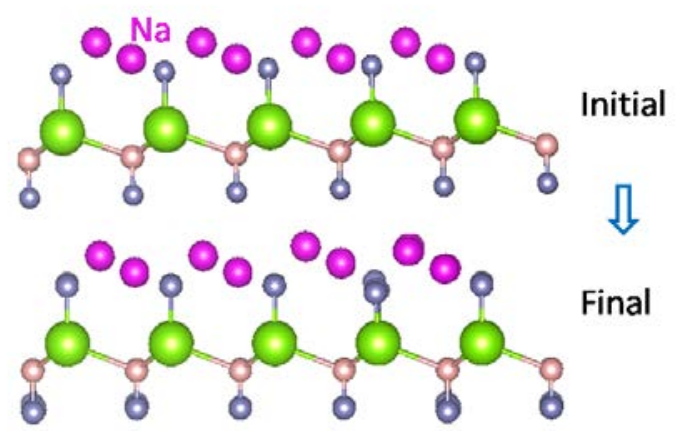

Fig. 6 Comparison of structure snapshots between the initial and final states for (a) the $\mathrm{Li}_{3} \mathrm{GaNH}_{2}$ and (b) the $\mathrm{Na}_{2} \mathrm{GaNH}_{2}$ system after 3 ps of AIMD simulation at $323 \mathrm{~K}$. 